\title{
QUASICONFORMAL GROUPS WITH SMALL DILATATION
}

\author{
M. JEAN MCKEMIE
}

\section{Introduction}

In this paper we consider groups of uniformly quasiconformal homeomorphisms acting on $\overline{\mathbf{R}}^{n}$. One method of constructing such a group is to conjugate a conformal, (i.e., Möbius) group by a quasiconformal mapping. A question raised by Gehring and Palka [GP] was whether this might not be the only way, that is, if each uniformly quasiconformal group is the quasiconformal conjugate of a conformal group. This question was answered affirmatively by Sullivan [S] and Tukia [T 1] for quasiconformal groups acting on subsets of $\overline{\mathbf{R}}^{2}$. Later Tukia [T 3] exhibited, for every $n>2$, a quasiconformal group acting on $\overline{\mathbf{R}}^{n}$ which is not isomorphic to, hence is not the quasiconformal conjugate of, a Möbius group. Tukia's example was modified by Martin [M] to yield discrete, non-elementary (i.e., with limit set containing more than two points) quasiconformal groups acting on $\overline{\mathbf{R}}^{n}, n \geqq 3$, which are not quasiconformally equivalent to conformal groups. More recently, Freedman and Skora [FS] have produced a discrete group $G$ of smooth quasiconformal mappings acting on $S^{3}$, where $G$ is not even topologically conjugate to a Möbius group. Since each of the quasiconformal groups mentioned above is a group with large dilatation, it was a matter of some interest to obtain a quasiconformal group with small dilatation which is not quasiconformally conjugate to a Möbius group. We modify Tukia's construction to produce such groups. We demonstrate that, for each $n \geqq 3$ and $K>1$, there is a $K$-quasiconformal group acting on $\overline{\mathbf{R}}^{n}$ which is not the quasiconformal conjugate of a Möbius group. Furthermore, we extend a second result of Tukia, in that we exhibit for each $n \geqq 3$ and $K>1$, a Jordan domain in $\overline{\mathbf{R}}^{n}$ which is homogeneous with respect to the action of a $K$-quasiconformal group and which is topologically, but not quasiconformally, equivalent to the open unit ball $B^{n}$. 


\section{Definitions and notation}

Let $X$ and $Y$ be metric spaces. An embedding $f: X \rightarrow Y$ is called bilipschitz (or more precisely, L-bilipschitz) if, for some $L \geqq 1$,

$$
\frac{|x-y|}{L} \leqq|f(x)-f(y)| \leqq L|x-y|
$$

for all $x, y \in X$, where we use euclidean notation $|x-y|$ to denote the distance between points $x$ and $y$ in an arbitrary metric space. An embedding $f: X \rightarrow Y$ is quasisymmetric (or $\eta$-quasisymmetric) if there is a homeomorphism $\eta:[0, \infty) \rightarrow[0, \infty)$ such that

$$
|f(x)-f(y)| \leqq \eta(t)|f(x)-f(v)|
$$

whenever $|x-y| \leqq t|x-v|$. If it is merely true that there is an $H \geqq 1$ so that

$$
|f(x)-f(y)| \leqq H|f(x)-f(v)|
$$

whenever $|x-y| \leqq|x-v|$, we say $f$ is a weakly H-quasisymmetric embedding. Clearly every $\eta$-quasisymmetric embedding is weakly $\eta(1)$-quasisymmetric. Consider the space $\mathscr{H}$ of all homeomorphisms $\eta:[0, \infty) \rightarrow[0, \infty)$. Let id denote the identity mapping of $[0, \infty)$ and for $s>0$, let $N($ id, $s)=\{\eta \in \mathscr{H}:|\eta(t)-t| \leqq s, 0 \leqq t \leqq 1 / s\}$. The sets $N($ id, $s)$ form a basis for the neighborhood system of the identity in the compact-open topology on $\mathscr{H}$. We say $f$ is $s$-quasisymmetric if $f$ is $\eta$-quasisymmetric for some $\eta$ in $N(\mathrm{id}, s)$. An embedding which is $\eta$-quasisymmetric with $\eta(t)=t$ is called 0-quasisymmetric. Our terminology here conforms to that in [TV 1, TV 2]. We denote the identity map on the space $X$ by $\mathrm{id}_{X}$. When the context is clear, we also write $\operatorname{id}_{X}$ for the embedding of $X$ into $Y$ given by $\operatorname{id}_{X}(x)=x$ for all $x \in X$.

If $G$ is a family of homeomorphisms of a metric space $X$ and if each $g \in G$ is $L$-bilipschitz, we say that $G$ is an $L$-Lipschitz family. If, in addition, $G$ forms a group under composition, we say $G$ is an L-Lipschitz group. We call $G$ a uniformly Lipschitz family (or group) if it is an $L$-Lipschitz family (or group) for some $L$.

If $U \subset \overline{\mathbf{R}}^{n}$ is open and $G$ is a group of self-homeomorphisms of $U$ such that each $g \in G$ is $K$-quasiconformal, we say that $G$ is a $K$-quasiconformal group. If every $g \in G$ is the restriction to $U$ of a Möbius transformation, we call $G$ a Möbius group. A uniformly quasiconformal group is a group which is $K$-quasiconformal for some $K$.

If $A: \mathbf{R}^{n} \rightarrow \mathbf{R}^{n}$ is a linear mapping, then we set

$$
|A|=\max _{|h|=1}|A h|, \quad l(A)=\min _{|h|=1}|A h|,
$$

and write $\operatorname{det} A$ for the determinant of $A$. Let $U \subset \mathbf{R}^{n}$ be open. If $f: U \rightarrow \mathbf{R}^{m}$ is differentiable at $x \in U$, the derivative of $f$ at $x$ is denoted $D f(x)$ and the Jacobian determinant of $f$ at $x$ is $J(x, f)$. 
The notation $B^{n}(x, r)$ indicates the open ball $\left\{y \in \mathbf{R}^{n}:|x-y|<r\right\}$ and we abbreviate $B^{n}=B^{n}(0,1)$. Similarly, $S^{n-1}(x, r)$ is the sphere $\left\{y \in \mathbf{R}^{n}:|x-y|=r\right\}$ and $S^{n-1}=S^{n-1}(0,1)$. By $I$ we mean the closed interval $[0,1]$ in $\mathbf{R}$, while $I^{n}=$ $[0,1]^{n}$, the $n$-dimensional unit cube. $\mathbf{C}$ is the complex plane, which we identify with $\mathbf{R}^{2}$. Depending on the context, we use either $x=x_{1}+i x_{2}$ or $x=\left(x_{1}, x_{2}\right)$ to denote $x \in \mathbf{R}^{2}$. The upper half plane in $\mathbf{C}$ is $\Omega_{0}=\{z \in \mathbf{C}: \operatorname{Im} z>0\}$ while $\Omega_{1}=$ $\{z \in \mathbf{C}: \operatorname{Im} z<0\}$, the lower half plane. We let $\overline{\mathbf{C}}=\mathbf{C} \cup\{\infty\}$ and $\overline{\mathbf{R}}^{n}=\mathbf{R}^{n} \cup\{\infty\}$. For $A \subset \overline{\mathbf{R}}^{n}, \partial A$ denotes the boundary of $A$ and $\bar{A}$ denotes the closure of $A$, both taken in $\overline{\mathbf{R}}^{n}$. We write dist $(A, B)$ for the euclidean distance between sets $A$ and $B$ in $\mathbf{R}^{n}$.

Let $U$ be a simply connected region in $\mathbf{C}$ which is not the whole plane. Then the hyperbolic metric $h_{U}$ on $U$ is defined by

$$
h_{U}\left(z_{1}, z_{2}\right)=\inf _{\gamma} \int_{\gamma} \lambda_{U}(z)|d z|,
$$

where $\lambda_{U}$ is the Poincare density in $U$ and the infimum is extended over all rectifiable paths $\gamma$ joining $z_{1}$ and $z_{2}$ in $U$. The Poincaré density $\lambda_{U}$ is given by

$$
\lambda_{U}(f(z))|D f(z)|=\frac{1}{\operatorname{Im}(z)},
$$

where $f$ is any conformal mapping of $\Omega_{0}$ onto $U$. In the case $U=\Omega_{i}, i=0$ or 1 , then

$$
\lambda_{U}(z)=\frac{1}{|\operatorname{Im}(z)|}=\frac{1}{\operatorname{dist}(z, \partial U)} .
$$

If $U$ is a proper subdomain in $\mathbf{R}^{n}$, the quasihyperbolic metric $q_{U}$ of $U$ is defined using the density $\varrho_{U}$,

For $x, y \in U$ we let

$$
\varrho_{U}(x)=\frac{1}{\operatorname{dist}(x, \partial U)}
$$

$$
q_{U}(x, y)=\inf _{\gamma} \int_{\gamma} \varrho_{U}(\zeta)|d \zeta|
$$

with the infimum being extended over all rectifiable paths $\gamma$ joining $x$ and $y$ in $U$. Note that if $U=\Omega_{i}, i=0$ or 1 , then $q_{U}=h_{U}$. We shall often write simply $q$ or $h$ in these cases.

\section{Extension of weakly $H$-quasisymmetric embeddings of $\mathbf{R}$ into $\mathbf{C}$}

In this section we establish an extension result we shall need in order to control the dilatations of the groups constructed in Section 5. We appeal to results of Tukia and Väisälä, as well as to a result of J. Vaaler and the author [MV].

An embedding $f: \mathbf{R} \rightarrow \mathbf{C}$ is normalized if $f(0)=0$ and $f(1)=1$. We now establish 
Theorem 1. Let $f: \mathbf{R} \rightarrow \mathbf{C}$ be a weakly H-quasisymmetric embedding. Then $f$ is $s$-quasisymmetric, where $s=s(H)$ can be chosen to satisfy $s \rightarrow 0$ as $H \rightarrow 1$.

Proof. By [TV 1, 2.16] $f$ is $\eta$-quasisymmetric for some homeomorphism $\eta$ depending only on $H$. Let $s(H)$ be the smallest $s$ so that $\eta \in N(\mathrm{id}, s)$, i.e., $s(H)$ is the smallest $s$ so that any weakly $H$-quasisymmetric $f: \mathbf{R} \rightarrow \mathbf{C}$ is $s$-quasisymmetric. Assume that $s \rightarrow 0$ as $H \rightarrow 1$. Then there exists a number $s \in(0,1 / 6]$, a sequence $H_{j} \downarrow 1$, and a sequence of embeddings $f_{j}: \mathbf{R} \rightarrow \mathbf{C}$ so that each $f_{j}$ is weakly $H_{j}$-quasisymmetric, but such that no $f_{j}$ is $2 s$-quasisymmetric. Appealing to [TV 2, 2.4] we can choose points $a_{j}, b_{j}, x_{j} \in \mathbf{R}$ with

for which

$$
\frac{\left|a_{j}-x_{j}\right|}{\left|b_{j}-x_{j}\right|}=t_{j} \in\left[s, \frac{1}{s}\right]
$$

$$
t_{j}^{*}=\frac{\left|f_{j}\left(a_{j}\right)-f_{j}\left(x_{j}\right)\right|}{\left|f_{j}\left(b_{j}\right)-f_{j}\left(x_{j}\right)\right|}>t_{j}+s .
$$

Performing auxiliary similarities we are free to assume that $a_{j}=1=f_{j}\left(a_{j}\right)$ and that $x_{j}=0=f_{j}\left(x_{j}\right)$. Consequently, we have

and

$$
\frac{1}{\left|b_{j}\right|}=t_{j} \in\left[s, \frac{1}{s}\right]
$$

$$
\frac{1}{\left|f_{j}\left(b_{j}\right)\right|}=t_{j}^{*}>t_{j}+s
$$

Now this gives $\left|b_{j}\right|=1 / t_{j} \in[s, 1 / s]$. We may pass to a subsequence and assume that $\left\{b_{j}\right\}_{j=1}^{\infty}$ converges to $b$ where $|b| \in[s, 1 / s]$. Each $f_{j}$ is normalized, so according to [TV 1, 3.4 and 3.7] we may further assume that $f_{j} \rightarrow f$ uniformly on compact subsets of $\mathbf{R}$, where $f$ is a quasisymmetric embedding of $\mathbf{R}$ into $\mathbf{C}$. Now for $x, y, v$ fixed in $\mathbf{R}$ with $|x-y| \leqq|v-y|$ we have

$$
\frac{\left|f_{j}(x)-f_{j}(y)\right|}{\left|f_{j}(v)-f_{j}(y)\right|} \leqq H_{j} .
$$

Hence, taking the limits, we obtain

$$
\frac{|f(x)-f(y)|}{|f(v)-f(y)|} \leqq 1 .
$$

Thus $f$ is weakly 1-quasisymmetric and normalized. Now a theorem in [MV] asserts that $f$ is the identity. We conclude that the subsequence $f_{j} \rightarrow \mathrm{id}_{\mathbf{R}}$ uniformly on compact subsets of $\mathbf{R}$. Therefore

$$
\lim _{j \rightarrow \infty} \frac{1}{\left|b_{j}\right|}=\frac{1}{|b|}=\frac{1}{|f(b)|}=\lim _{j \rightarrow \infty} \frac{1}{\left|f_{j}\left(b_{j}\right)\right|}=\lim _{j \rightarrow \infty} t_{j}^{*} .
$$


However, this implies that

$$
\frac{1}{|b|}=\lim _{j \rightarrow \infty} t_{j}^{*} \geqq \lim _{j \rightarrow \infty} t_{j}+s=\lim _{j \rightarrow \infty} \frac{1}{\left|b_{j}\right|}+s=\frac{1}{|b|}+s .
$$

Since $s>0$, this is a contradiction. We conclude that $s(H)$ can be selected so as to ensure that $s(H) \rightarrow 0$ as $H \rightarrow 1$.

Corollary 2. Let $f: \mathbf{R} \rightarrow \mathbf{C}$ be a weakly H-quasisymmetric embedding. Then $f$ has an extension to a weakly $K$-quasisymmetric and $K$-quasiconformal homeomorphism $F: \mathbf{C} \rightarrow \mathbf{C}$, where $K=K(H)$ can be chosen so that $K \rightarrow 1$ as $H \rightarrow 1$.

Proof. By Theorem $1 f$ is $s$-quasisymmetric, where $s=s(H)$ and $s \rightarrow 0$ as $H \rightarrow 1$. Using [TV 2, 5.4] we see that there exists $s_{0}>0$ so that, if $f$ is $s$-quasisymmetric with $s<s_{0}$, then $f$ has a $\sigma_{1}$-quasisymmetric extension $F: \mathbf{C} \rightarrow \mathbf{C}$. Here $\sigma_{1}=\sigma_{1}(s)$ may be chosen so that $\sigma_{1} \rightarrow 0$ as $s \rightarrow 0$. Choose $H_{0}$ so that for all $H<H_{0}, s(H)<s_{0}$. Then for $H<H_{0}, \quad F$ is $\sigma_{1}$-quasisymmetric, where $\sigma_{1}=\sigma_{1}(H)=\sigma_{1}(s(H)) \rightarrow 0$, as $H \rightarrow 1$. By [TV2, 2.6], $F$ is $K$-quasiconformal and weakly $K$-quasisymmetric for $K=K\left(\sigma_{1}\right)$ satisfying $K\left(\sigma_{1}\right) \rightarrow 1$ as $\sigma_{1} \rightarrow 0$. In other words, we conclude that $f$ has a weakly $K$-quasisymmetric and $K$-quasiconformal extension $F$ to $\mathbf{C}$, where $K=$ $K(H)=K\left[\sigma_{1}(H)\right]$ may be chosen to satisfy $K \rightarrow 1$ as $H \rightarrow 1$.

\section{The embeddings $f$ and $F_{f}$}

Fix an integer $n \geqq 1$. We construct, corresponding to $n$, an embedding $f: \mathbf{R} \rightarrow \mathbf{C}$. If we wish to emphasize the dependence of $f$ on $n$, we write $f=f_{(n)}$. To begin, we define a sequence of embeddings $f_{j}$ of $I=[0,1]$ into C. Restricted to $I, f$ will just be the limit of this sequence of embeddings. Set

$$
s_{n}=\frac{1}{4}\left(1+3^{-n}\right) \text {. }
$$

We define the embeddings $f_{j}, j=0,1, \ldots$, inductively. Take $f_{0}=\mathrm{id}_{I}$. Subdivide $I$ into four segments $I_{k}^{1}$, where $I_{k}^{1}=[(k-1) / 4, k / 4]$ for $k=1,2,3,4$. Note that $I_{k}^{1}$ is of length $1 / 4$. We define $f_{1}$ so that $\left.f_{1}\right|_{k} ^{1}$ is affine and $f_{1}(I)$, which we denote $J^{1}$, is an arc consisting of four line segments of length $s_{n}$. We specify the images of the endpoints of $I_{k}^{1}$ for $k=1,2,3,4$, as follows:

$$
\begin{gathered}
f_{1}(0)=0, \quad f_{1}\left(\frac{1}{4}\right)=s_{n}, \quad f_{1}\left(\frac{1}{2}\right)=\frac{1}{2}\left(1+i 3^{-n / 2}\right), \\
f_{1}\left(\frac{3}{4}\right)=1-s_{n}, \quad \text { and } f_{1}(1)=1 .
\end{gathered}
$$

See Figure 1.

We proceed to the inductive definition. We assume $f_{j}$ has been defined on $I=\bigcup_{l=1}^{4^{j}} I_{l}^{j}$, where $I_{l}^{j}=\left[(l-1) / 4^{j}, l / 4^{j}\right]$. Define $f_{j+1}$ so that $\left.f_{j+1}\right|_{I_{l}^{j+1}}$ is affine for 

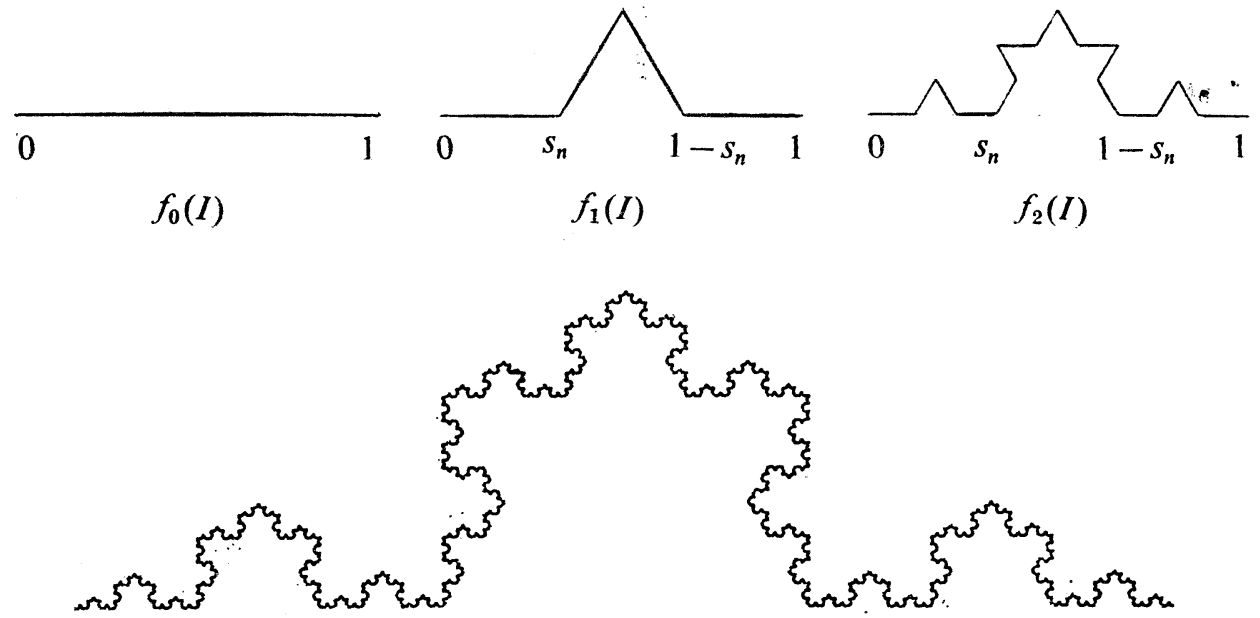

$J^{*}$ for $n=1$
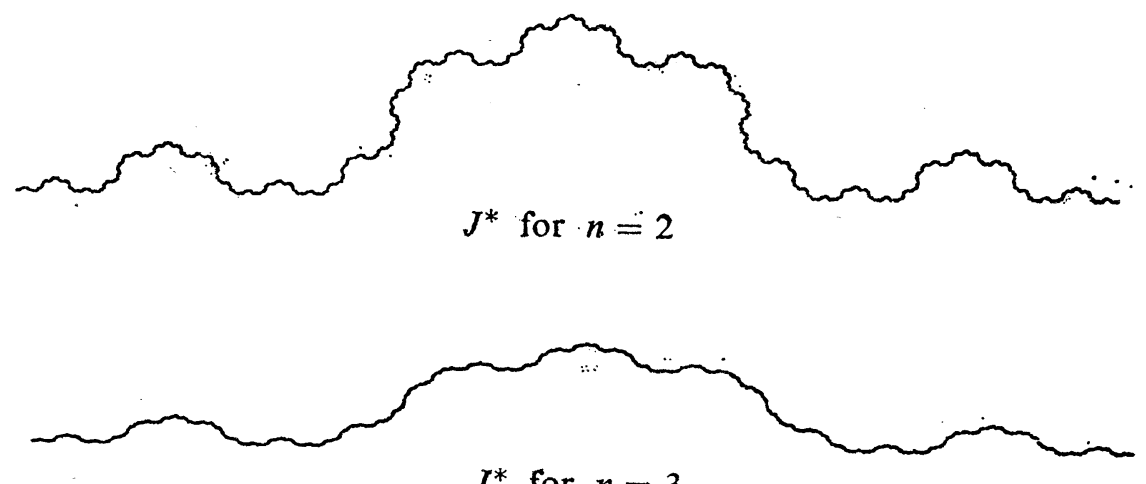

$J^{*}$ for $n=3$.

Figure 1

$l=1,2, \ldots, 4^{j+1}$ and so that for $l=1, \ldots, 4^{j}$

$$
\begin{aligned}
& f_{j+1}\left(\frac{l-1}{4^{j}}\right)=f_{j}\left(\frac{l-1}{4^{j}}\right) \\
& f_{j+1}\left(\frac{4(l-1)+1}{4^{j+1}}\right)=f_{j}\left(\frac{l-1}{4^{j}}\right)+s_{n}\left[f_{j}\left(\frac{l}{4^{j}}\right)-f_{j}\left(\frac{l-1}{4^{j}}\right)\right] \\
& f_{j+1}\left(\frac{4(l-1)+2}{4^{j+1}}\right)=\frac{1}{2}\left[f_{j}\left(\frac{l-1}{4^{j}}\right)+f_{j}\left(\frac{l}{4^{j}}\right)+i 3^{-n / 2}\left(f_{j}\left(\frac{l}{4^{j}}\right)-f_{j}\left(\frac{l-1}{4^{j}}\right)\right)\right], \\
& f_{j+1}\left(\frac{4(l-1)+3}{4^{j+1}}\right)=f_{j}\left(\frac{l}{4^{j}}\right)-s_{n}\left[f_{j}\left(\frac{l}{4^{j}}\right)-f_{j}\left(\frac{l-1}{4^{j}}\right)\right],
\end{aligned}
$$


and

$$
f_{j+1}\left(\frac{4(l-1)+4}{4^{j+1}}\right)=f_{j+1}\left(\frac{l}{4^{j}}\right)=f_{j}\left(\frac{l}{4^{j}}\right) .
$$

Then $f_{j+1}\left(I_{1}^{j}\right)=\left(s_{n}\right)^{j} f_{1}(I)$, and $f_{j+1}\left(I_{l}^{j}\right)$ for $l=1,2, \ldots, 4^{j}$ is a rotated, and perhaps translated, copy of $\left(s_{n}\right)^{j} f_{1}(I)$. If we denote $f_{j}(I)$ by $J^{j}$, we see that the dilate $s_{n} J^{j}$ is a subarc of $J^{j+1}$. We note that $\max _{x \in I}\left|f_{j+1}(x)-f_{j}(x)\right| \leqq\left(s_{n} / 2\right)^{j} 3^{-n / 2}$ which implies that $\left\{f_{j}\right\}$ is uniformly Cauchy on $I$. Hence $\left\{f_{j}\right\}$ converges to $f$ uniformly on $I$, where $f: I \rightarrow \mathbf{C}$ is a continuous mapping. As $f$ is easily seen to be injective, we conclude that $f$ is an embedding. Write $f(I)=J^{*}$. Some examples of $J^{*}$ are shown in Figure 1, along with examples of $f_{j}(I)$.

By the manner in which we defined $f_{j}$, we see that $s_{n} J^{*}$ is a subarc of $J^{*}$ and that $J^{*}$ is a subarc of $\left(1 / s_{n}\right) J^{*}$. Set

$$
J=\bigcup_{l \geqq 0}\left(\frac{1}{s_{n}}\right)^{l}\left[J^{*} \cup\left(-J^{*}\right)\right] .
$$

By construction, for $l \geqq 0$ and $x$ satisfying $0 \leqq x \leqq 4^{-l}$, we have

$$
f\left(4^{l} x\right)=\left(\frac{1}{s_{n}}\right)^{l} f(x) .
$$

We extend $f$ to $\mathbf{R}$ by setting

$$
f\left( \pm 4^{l} x\right)= \pm\left(\frac{1}{s_{n}}\right)^{l} f(x),
$$

for $x \in I$. The resulting map, still called $f$, is a homeomorphism of $\mathbf{R}$ onto $J$ and satisfies

$$
\max _{x \in I}|f(x)-x| \leqq\left(\frac{3}{4}\right) 3^{-n / 2}
$$

For $n \geqq 1$ a fixed integer, we define $0<\alpha_{n}<1$ by

$$
\alpha_{n}=\frac{\log \left(\frac{1}{4}\left(1+3^{-n}\right)\right)}{\log \frac{1}{4}},
$$

and we set $g_{n}(x)=x^{\alpha_{n}}$ for $x \in[0,2]$. A computation yields

Lemma 3. The mappings $g_{n}$ converge uniformly to the identity mapping on $[0,2]$ as $n \rightarrow \infty$.

Next we establish some significant estimates.

Lemma 4. Let $0<\varepsilon<1$. There exists $N \geqq 1$ so that $f=f_{(n)}$ satisfies

$$
1-\varepsilon \leqq \frac{|f(x)-f(y)|}{|x-y|^{\alpha_{n}}} \leqq \frac{1}{1-\varepsilon}
$$

for all $x, y \in[-1,1]$, whenever $n \geqq N$. 
Proof. Let $x, y \in[-1,1]$ with $|x-y| \geqq 4^{-4}$. Without loss of generality, assume $y>x$. By (3) and Lemma 3 we may find $N$ so that $f=f_{(n)}$ satisfies

$$
\max _{x \in[-1,1]}|f(x)-x|<\frac{\varepsilon}{4^{8}}
$$

and so that for all $x, y \in[-1,1]$

$$
|| x-\left.y\right|^{\alpha_{n}}-|x-y| \mid<\frac{\varepsilon}{4^{8}}
$$

whenever $n>N$. Then

$$
\begin{aligned}
\frac{|f(x)-f(y)|}{|x-y|^{\alpha_{n}}} & \leqq \frac{|f(x)-x|+|x-y|+|y-f(y)|}{|x-y|^{\alpha_{n}}} \\
& \leqq \frac{|x-y|^{\alpha_{n}}+\varepsilon / 4^{8}}{|x-y|^{\alpha_{n}}}+\frac{2 \varepsilon / 4^{8}}{|x-y|^{\alpha_{n}}} \\
& \leqq 1+\frac{3 \varepsilon}{4^{8}|x-y|^{\alpha_{n}}} \\
& \leqq \frac{1}{1-\varepsilon} .
\end{aligned}
$$

By the triangle inequality,

$$
|y-f(y)|+|f(y)-f(x)|+|f(x)-x| \geqq|x-y| .
$$

This implies that

$$
\begin{aligned}
\frac{|f(x)-f(y)|}{|x-y|^{\alpha_{n}}} & \geqq \frac{|x-y|-\left(2 \varepsilon / 4^{8}\right)}{|x-y|^{\alpha_{n}}} \\
& \geqq \frac{|x-y|^{\alpha_{n}}-\left(3 \varepsilon / 4^{8}\right)}{|x-y|^{\alpha_{n}}} \\
& \geqq 1-\varepsilon .
\end{aligned}
$$

We have shown that (4) holds for all $x, y \in[-1,1]$ with $|x-y| \geqq 4^{-4}$. We must also show that it holds for $|x-y|<4^{-4}$. We consider two situations: Case (a): $x$ and $y$ are both in $I$ (or, what is the same, by symmetry, $x$ and $y$ both in $-I$ ); Case (b): $x \in-I$ and $y \in I$. We may again assume that $x<y$.

Assume we are in Case (a). Then $x \in I_{l}^{4}$ for some $l$. Since $|x-y|<4^{-4}$, either $y \in I_{l}^{4}$ as well, or $y \in I_{l+1}^{4}$. Suppose first that $y \in I_{l+1}^{4}$. At the fifth stage of our construction, $I_{l}^{4}$ is subdivided into 4 segments, say

$$
I_{l}^{4}=I_{m}^{5} \cup I_{m+1}^{5} \cup I_{m+2}^{5} \cup I_{m+3}^{5} .
$$

Similarly, $I_{l+1}^{4}$ consists of the next four segments. The following situations might 
conceivably arise:

(i) $x \in I_{m+3}^{5}$ and $y \in \bigcup_{j=4}^{7} I_{m+j}^{5}$.

(ii) $x \in I_{m+2}^{5}$ and $y \in \bigcup_{j=4}^{6} I_{m+j}^{5}$.

(iii) $x \in I_{m+1}^{5}$ and $y \in \bigcup_{j=4}^{5} I_{m+j}^{5}$.

(iv) $x \in I_{m}^{5}$ and $y \in I_{m+4}^{5}$.

Only in the situation $x \in I_{m+3}^{5}$ and $y \in I_{m+4}^{5}$ do $x$ and $y$ lie in adjacent segments of length $4^{-5}$. Suppose any of the other possibilities occur. Then $|x-y| \geqq 4^{-5}$. Now, by the construction, $f_{4}\left(I_{l}^{4} \cup I_{l+1}^{4}\right)$ is an arc consisting of two line segments, each of length $\left(s_{n}\right)^{4}$. Furthermore, $f_{4}\left(I_{l}^{4} \cup I_{l+1}^{4}\right)$ is a rotated and translated copy of $f_{4}\left(I_{1}^{4} \cup I_{2}^{4}\right)$, $f_{4}\left(I_{2}^{4} \cup I_{3}^{4}\right)$, or $f_{4}\left(I_{3}^{4} \cup I_{4}^{4}\right)$. Since $f_{4}$ is affine on each $I_{m}^{4}$, we may thus find points $x_{1}$ and $y_{1}$ in $I_{1}^{3}=\bigcup_{j=1}^{4} I_{j}^{4}$ with $\left|x_{1}-y_{1}\right|=|x-y|$ and $\left|f_{4}\left(x_{1}\right)-f_{4}\left(y_{1}\right)\right|=\left|f_{4}(x)-f_{4}(y)\right|$. Hence, we might as well consider $x$ and $y$ in $I_{1}^{3}$. Recall that $f_{4}\left(I_{1}^{3}\right)=\left(s_{n}\right)^{3} f_{1}(I)$ and $f\left(I_{1}^{3}\right)=\left(s_{n}\right)^{3} f(I)$. Thus there exist $x^{*}, y^{*} \in I$ with $\left|x^{*}-y^{*}\right|=4^{3}|x-y| \geqq 4^{-4}$ and $|f(x)-f(y)|=\left(s_{n}\right)^{3}\left|f\left(x^{*}\right)-f\left(y^{*}\right)\right|$. This implies that

$$
\frac{|f(x)-f(y)|}{|x-y|^{\alpha_{n}}}=\frac{\left(s_{n}\right)^{3}\left|f\left(x^{*}\right)-f\left(y^{*}\right)\right|}{\left(4^{-3}\left|x^{*}-y^{*}\right|\right)^{\alpha_{n}}}=\frac{\left|f\left(x^{*}\right)-f\left(y^{*}\right)\right|}{\left|x^{*}-y^{*}\right|^{\alpha_{n}}} .
$$

Since (4) holds for the final expression, it must hold for the first.

We have not dealt with the case $x \in I_{m+3}^{5}$ and $y \in I_{m+4}^{5}$ which occured in (i) or the case $x \in I_{l}^{4}$ and $y \in I_{l}^{4}$. We handle these with one argument as follows. In both instances there is a largest integer $k$ so that for some $h, x \in I_{h}^{k}$ and $y \in I_{h+1}^{k}$. At the next stage, at least one interval of length $4^{-(k+1)}$ must lie between $x$ and $y$. Again, the image of $I_{h}^{k} \cup I_{h+1}^{k}$ is a rotated and translated copy of the image of $I_{1}^{k} \cup I_{2}^{k}, I_{2}^{k} \cup I_{3}^{k}$, or $I_{3}^{k} \cup I_{4}^{k}$, so we may consider $x$ and $y$ in $I_{1}^{k-1}$. Since $f\left(I_{k}^{k-1}\right)=\left(s_{n}\right)^{k-1} f(I)$, there are $x^{*}, y^{*} \in I$ with $\left|x^{*}-y^{*}\right|=4^{k-1}|x-y|>4^{-4}$ and $|f(x)-f(y)|=\left(s_{n}\right)^{k-1}\left|f\left(x^{*}\right)-f\left(y^{*}\right)\right|$. Consequently,

$$
\frac{|f(x)-f(y)|}{|x-y|^{\alpha_{n}}}=\frac{\left(s_{n}\right)^{k-1}\left|f\left(x^{*}\right)-f\left(y^{*}\right)\right|}{\left(4^{-(k-1)}\left|x^{*}-y^{*}\right|\right)^{\alpha_{n}}}=\frac{\left|f\left(x^{*}\right)-f\left(y^{*}\right)\right|}{\left|x^{*}-y^{*}\right| \alpha_{n}} .
$$

We see that (4) holds for the last term, thus holds for the initial term as well.

Finally, consider Case (b). Now $x \in-I$ and $y \in I$. Then $x \in-I_{1}^{4}$ and $y \in I_{1}^{4}$, since $|x-y|<4^{-4}$. At the fifth subdivision of $-I \cup I$ either there is a segment of length $4^{-5}$ lying between $x$ and $y$ or $x \in-I_{1}^{5}$ and $y \in I_{1}^{5}$. If $|x-y| \geqq 4^{-5}$, then we have $f\left(-I_{1}^{4} \cup I_{1}^{4}\right)=\left(s_{n}\right)^{3} f(-I \cup I)$. Hence there are $x^{*}$ and $y^{*} \in-I \cup I$ with $\left|x^{*}-y^{*}\right|=4^{3}|x-y|>4^{-4}$, and

$$
\frac{|f(x)-f(y)|}{|x-y|^{\alpha_{n}}}=\frac{\left(s_{n}\right)^{3}\left|\left(x^{*}\right)-f\left(y^{*}\right)\right|}{\left(4^{-3}\left|x^{*}-y^{*}\right|\right)^{\alpha_{n}}}=\frac{\left|f\left(x^{*}\right)-f\left(y^{*}\right)\right|}{\left|x^{*}-y^{*}\right|^{\alpha_{n}}} .
$$

Again, (4) holds for the last expression so it must also be valid for the first. 
If $x \in-I_{1}^{5}$ and $y \in I_{1}^{5}$, then a similar argument leads to a largest $k$ so that $x \in-I_{1}^{k}$ and $y \in I_{1}^{k}$. The same argument is made using $f\left(-I_{1}^{k} \cup I_{1}^{k}\right)=\left(s_{n}\right)^{k-1} f(-I \cup I)$ and the result follows.

Thus, for all $x, y \in[-1,1]$ we have

provided that $n>N$.

$$
1-\varepsilon \leqq \frac{|f(x)-f(y)|}{|x-y|^{\alpha_{n}}} \leqq \frac{{ }^{\prime} 1}{1-\varepsilon},
$$

We next extend this result to the entire real line.

Corollary 5. Let $0<\varepsilon<1$. There exists $N \geqq 1$ so that $f=f_{(n)}$ satisfies

$$
1-\varepsilon \leqq \frac{|f(x)-f(y)|}{|x-y|^{\alpha_{n}}} \leqq \frac{1}{1-\varepsilon}
$$

for all $x, y \in \mathbf{R}$, whenever $n>N$.

Proof. Using Lemma 4, choose $N$ so that the estimate above holds for all $x, y \in[-1,1]$ whenever $n>N$. Suppose $x, y \notin[-1,1]$. We may assume $|x| \leqq|y|$. Then for some $k \geqq 1$, we have $x^{*}, y^{*} \in[-1,1]$ with $y=4^{k} y^{*}, x=4^{k} x^{*}$ and $f(y)=$ $\left(1 / s_{n}\right)^{k} f\left(y^{*}\right), f(x)=\left(1 / s_{n}\right)^{k} f\left(x^{*}\right)$. So

$$
\frac{|f(x)-f(y)|}{|x-y|^{\alpha_{n}}}=\frac{\left(1 / s_{n}\right)^{k}\left|f\left(x^{*}\right)-f\left(y^{*}\right)\right|}{\left(4^{k}\left|x^{*}-y^{*}\right|\right)^{\alpha_{n}}}=\frac{\left|f\left(x^{*}\right)-f\left(y^{*}\right)\right|}{\left|x^{*}-y^{*}\right|^{\alpha_{n}}} .
$$

Hence (4) holds for $f$ on $\mathbf{R}$.

It is a direct consequence of (4) that $f$ is weakly $1 /(1-\varepsilon)^{2}$-quasisymmetric.

We summarize the foregoing in

Lemma 6. Given $H>1$ there exists a weakly $H$-quasisymmetric embedding $f: \mathbf{R} \rightarrow \mathbf{C}$ satisfying

$$
\frac{1}{H} \leqq \frac{|f(x)-f(y)|}{|x-y|^{\alpha}} \leqq H
$$

for all $x$ and $y$, where $\alpha=\alpha(H) \in(0,1)$ can be chosen to satisfy $\alpha(H) \rightarrow 1$ as $H \rightarrow 1$.

Proof. Given $H$, choose $0<\varepsilon<1$ so that $1 /(1-\varepsilon)=H^{1 / 2}<H$. Applying Corollary 5 for this $\varepsilon=\varepsilon(H)$, we may fix $n=n(H)>1 /(H-1)$ so that $f=f_{(n)}$ satisfies (5) with $\alpha=\alpha_{n}$. Then $\alpha_{n}=\alpha(H) \rightarrow 1$ as $H \rightarrow 1$, and $f$ is seen to be weakly $H$-quasisymmetric.

The Tukia extension $F_{f}$. Fix $H>1$. Using Lemma 6 we find a weakly $H$-quasisymmetric embedding $f: \mathbf{R} \rightarrow \mathbf{C}$ satisfying 5. By Corollary 2, we may extend $f$ to $F$, a weakly $K_{1}(H)$-quasisymmetric and $K_{1}(H)$-quasiconformal homeomorphism of C to itself, where $K_{1}(H) \rightarrow 1$ as $H \rightarrow 1$.

We actually desire a modified extension, one of a particular form. This extension is originally due to Tukia [T 2]. Remember that $f$, hence $F$, is normalized. 
Let $C_{0}$ be the component of $\mathbf{C} \backslash f(\mathbf{R})$ such that the triple $(0,1, \infty)$ describes the positive orientation of $\partial C_{0}$ with respect to $C_{0}$. Denote the remaining component by $C_{1}$. Let $A_{f}$ be the homeomorphism mapping $\bar{\Omega}_{0}$ onto $\bar{C}_{0}$ which is conformal in $\Omega_{0}$ and fixes 0,1 , and $\infty$. Note that $f(\mathbf{R})=F(\mathbf{R})$ is a $K_{1}(H)$-quasicircle.

Consider the mapping $\left.A_{f}^{-1} \circ F\right|_{\Omega_{0}}$. Since $A_{f}^{-1} \circ F\left(\Omega_{0}\right)=A_{f}^{-1}\left(C_{0}\right)=\Omega_{0}$, we see that $A_{f}^{-1} \circ F$ is a $K_{1}(H)$-quasiconformal self-mapping of $\Omega_{0}$. By a well known theorem of Beurling and Ahlfors [BA], $g=\left.A_{f}^{-1} \circ F\right|_{\mathbf{R}}=A_{f}^{-1} \circ f$ is a $k$-quasisymmetric self-mapping of $\mathbf{R}$, where $k=k\left(K_{1}\right)=k(H)$ can be chosen so that $k(H) \rightarrow 1$ as $H \rightarrow 1$. Then [L, Theorem 5.2] asserts that there is a $K_{2}(k)$-quasiconformal selfmapping $B_{g}$ of $\Omega_{0}$ which extends $g$. Furthermore $K_{2}(H)=K_{2}(k(H))$ can be chosen so that $K_{2}(H) \rightarrow 1$ as $H \rightarrow 1$. We remark that $B_{g}$ is a diffeomorphism on $\Omega_{0}$, and is given by

$$
B_{g}(x+i y)=\frac{1}{2}[\alpha(x, y)+\beta(x, y)]+i[\alpha(x, y)-\beta(x, y)],
$$

where $\alpha(x, y)=\int_{0}^{1} g(x+t y) d t$ and $\beta(x, y)=\int_{0}^{1} g(x-t y) d t$. We refer to $B_{g}$ as the Beurling-Ahlfors extension of $g$. We also note that the partial derivatives of $\alpha$ and $\beta$ satisfy

$$
\left\{\begin{array}{l}
\alpha_{x}(x, y)=\frac{1}{y}[g(x+y)-g(x)], \quad \beta_{x}(x, y)=\frac{1}{y}[g(x)-g(x-y)], \\
\alpha_{y}(x, y)=\frac{1}{y}[g(x+y)-\alpha(x, y)], \quad \beta_{y}(x, y)=\frac{1}{y}[g(x-y)-\beta(x, y)] .
\end{array}\right.
$$

Now, set $F_{f}=A_{f} \circ B_{g}$. This definition is due to Tukia [T 2, p. 92], hence we refer to $F_{f}$ as the Tukia extension of $f$. (In [T 2], $B_{g}=1 / 2[\alpha+\beta]+i / 2[\alpha-\beta]$. All of the results we shall need from [T 2] remain valid despite this difference in the imaginary part of $B_{g}$.) We observe that $F_{f}: \Omega_{0} \rightarrow C_{0}$ is $K_{2}(H)$-quasiconformal.

We define $F_{f}$ on $\Omega_{1}$ in an analogous manner to obtain $F_{f}: \mathbf{C} \rightarrow \mathbf{C}$, a $K_{2}(H)$ quasiconformal homeomorphism with $K_{2}(H) \rightarrow 1$ as $H \rightarrow 1$. Note that $F_{f}$ is a diffeomorphism on $\mathbf{C} \backslash \mathbf{R}$.

We now establish several lemmas. We remark that Tukia has proven similar lemmas in [T 3], but made no attempt to obtain estimates of the size of the constants involved. Since we wish to control the dilatation of the groups we shall construct, we include the proofs of our lemmas to demonstrate that the constants can, in fact, be controlled.

We begin with

Lemma 7. Let $f$ be a weakly $H$-quasisymmetric embedding of $\mathbf{R}$ into $\mathbf{C}$ satisfying the conclusion of Lemma 6, let $\alpha=\alpha(H)$ as in Lemma 6 , let $F_{f}$ be the Tukia extension of $f$, and let $J=f(\mathbf{R})=F_{f}(\mathbf{R})$. Then there is a constant $M \geqq 1$ such that the double inequality

$$
\frac{|v|^{\alpha}}{M} \leqq \operatorname{dist}\left(F_{f}(u+i v), J\right) \leqq M|v|^{\alpha}
$$


holds for all $u, v \in \mathbf{R}$. Furthermore, $M=M(H)$ can be chosen so that $M(H) \rightarrow 1$ as $H \rightarrow 1$.

Proof. Since $F_{f}$ is weakly $K_{2}$-quasisymmetric on $\mathrm{C}$, where $K_{2}=K_{2}(H) \rightarrow 1$ as $H \rightarrow 1$, we have

$$
\left|F_{f}(z)-F_{f}(w)\right| \leqq K_{2}\left|F_{f}(z)-F_{f}(t)\right|
$$

whenever $|z-w| \leqq|z-t|$. Hence

$$
\begin{aligned}
\operatorname{dist}\left(F_{f}(u+i v), J\right) & \leqq\left|F_{f}(u+i v)-F_{f}(u)\right| \\
& \leqq K_{2}\left|F_{f}(u+v)-F_{f}(u)\right| \\
& =K_{2}|f(u+v)-f(u)| \\
& \leqq K_{2} H|v|^{\alpha},
\end{aligned}
$$

by (5).

On the other hand, let $z=f(x) \in J$ be a point with $\operatorname{dist}\left(F_{f}(u+i v), J\right)=$ $\left|F_{f}(u+i v)-z\right|$. Then $|(u+i v)-x| \geqq|(u+i v)-u|$, whence

$$
\begin{aligned}
\operatorname{dist}\left(F_{f}(u+i v), J\right) & =\left|F_{f}(u+i v)-z\right| \\
& =\left|F_{f}(u+i v)-F_{f}(x)\right| \\
& \geqq \frac{1}{K_{2}}\left|F_{f}(u+i v)-F_{f}(u)\right| \\
& =\frac{1}{K_{2}}\left|F_{f}(u+i v)-f(u)\right| \\
& \geqq\left(\frac{1}{K_{2}}\right)^{2}|f(u+v)-f(u)| \\
& \geqq\left(\frac{1}{K_{2}}\right)^{2}\left(\frac{1}{H}\right)|v|^{\alpha},
\end{aligned}
$$

again by (5). Hence (7) holds with $M(H)=H K_{2}^{2}$, and thus $M(H) \rightarrow 1$ as $H \rightarrow 1$.

We next have:

Lemma 8. Let $f$ be a normalized weakly $H$-quasisymmetric embedding of $\mathbf{R}$ into C. Let $F_{f}$ be its Tukia extension. Then there is a constant $L_{0} \geqq 1$ so that

$$
\frac{1}{L_{0}} \leqq l\left(D F_{f}\left(\frac{1}{2}+\frac{i}{2}\right)\right) \leqq\left|D F_{f}\left(\frac{1}{2}+\frac{i}{2}\right)\right| \leqq L_{0} .
$$

Furthermore, $L_{0}=L_{0}(H)$ may be chosen so that $L_{0}(H) \rightarrow 1$ as $H \rightarrow 1$.

Proof. The existence of $L_{0}$ follows from [T 2, p. 93]. We need only show that we may choose the constant so that $L_{0}(H) \rightarrow 1$ as $H \rightarrow 1$.

Suppose that $L_{0}(H)$ cannot be so chosen as to approach 1 as $H \rightarrow 1$. Then there is $\delta>0$ and a sequence of maps $f_{m}$ which are normalized weakly $H_{m}$-quasi- 
symmetric embeddings of $\mathbf{R}$ into $\mathbf{C}$ with $H_{m} \rightarrow 1$ such that either

$$
\left|D F_{f_{m}}\left(\frac{1}{2}+\frac{i}{2}\right)\right|>1+\delta
$$

for all $m$, or

$$
l\left(D F_{f_{m}}\left(\frac{1}{2}+\frac{i}{2}\right)\right)<1-\delta
$$

for all $m$.

Assume first that $(*)$ holds. The sequence $\left\{f_{m}\right\}$ is a compact family by [TV 1 , p. 109]. By passing to a subsequence we are free to assume $f_{m} \rightarrow f_{\infty}$, where $f_{\infty}$ is normalized and weakly 1-quasisymmetric. We conclude that $f_{\infty}(x)=x$ for all $x$ in $\mathbf{R}$ [MV, Corollary 2]. Note that $f_{m}(\mathbf{R})=F_{f_{m}}(\mathbf{R})$ is a $K\left(H_{m}\right)$-quasicircle, where by construction $K\left(H_{m}\right) \rightarrow 1$ as $H_{m} \rightarrow 1$, or, equivalently, as $m \rightarrow \infty$. It follows that the corresponding mappings $A_{f_{m}}$ are conformal homeomorphisms for which we may choose $\left[K\left(H_{m}\right)\right]^{2}$-quasiconformal extensions to $\mathbf{C}$ (for which we retain the notation $\left.A_{f_{m}}\right)$. According to $[\mathrm{V}, 20.5]$ the family $\left\{A_{f_{m}}\right\}$ is a normal family. By passing to a further subsequence, we may assume $A_{f_{m}} \rightarrow A$ uniformly on compact subsets of $\mathbf{C}$. We infer that $A$ is a 1-quasiconformal, normalized self-mapping of $\mathbf{C}$, whence $A$ is the identity mapping. Consequently, $A_{f_{m}}^{-1} \circ f_{m}$ converges to the identity mapping on R. Set $h_{m}=A_{f_{m}}^{-1} \circ f_{m}$ and $h_{\infty}=A^{-1} \circ f_{\infty}=\mathrm{id}_{\mathbf{R}}$. We see that

$$
B_{h_{m}}\left(\frac{1}{2}+\frac{i}{2}\right) \rightarrow B_{h_{\infty}}\left(\frac{1}{2}+\frac{i}{2}\right)=\frac{1}{2}+\frac{i}{2},
$$

where $B_{h_{m}}$ denotes the Beurling-Ahlfors extension of $h_{m}$. We observe that, as $m \rightarrow \infty$,

$$
F_{f_{m}}=A_{f_{m}} \circ B_{h_{m}} \rightarrow F_{f_{\infty}}=\left.i d\right|_{\mathbf{C}}
$$

where the convergence is uniform on compact subsets of $\mathbf{C}$. Using (6) and the fact that $\left|D B_{h}(z)\right|=\left|\partial B_{h}(z)\right|+\left|\bar{\partial} B_{h}(z)\right|$, we infer that

$$
\lim _{m \rightarrow \infty}\left|D B_{h_{m}}\left(\frac{1}{2}+\frac{i}{2}\right)\right|=\left|D B_{h_{\infty}}\left(\frac{1}{2}+\frac{i}{2}\right)\right|=1 .
$$

Because the mappings $A_{f_{m}}$ are conformal in $\Omega_{0}$ and converge uniformly on compact subsets of $\mathbf{C}$ to $\mathrm{id}_{\mathbf{C}}$, we have

$$
\begin{aligned}
\left|D F_{f_{m}}\left(\frac{1}{2}+\frac{i}{2}\right)\right| & =\max _{|h|=1}\left|D F_{f_{m}}\left(\frac{1}{2}+\frac{i}{2}\right) \cdot h\right| \\
& =\max _{|h|=1}\left|D A_{f_{m}}\left(B_{h_{m}}\left(\frac{1}{2}+\frac{i}{2}\right)\right) \cdot D B_{h_{m}}\left(\frac{1}{2}+\frac{i}{2}\right) \cdot h\right| \\
& =\left|A_{f_{m}}^{\prime}\left(B_{h_{m}}\left(\frac{1}{2}+\frac{i}{2}\right)\right)\right|\left|D B_{h_{m}}\left(\frac{1}{2}+\frac{i}{2}\right)\right|,
\end{aligned}
$$


and where $A^{\prime}$ denotes the complex derivative of $A$. Now

Therefore,

$$
\left|A_{f_{m}}^{\prime}\left(B_{h_{m}}\left(\frac{1}{2}+\frac{i}{2}\right)\right)\right| \rightarrow\left|A^{\prime}\left(\frac{1}{2}+\frac{i}{2}\right)\right|=1 .
$$

This means

$$
\lim _{m \rightarrow \infty}\left|D F_{f_{m}}\left(\frac{1}{2}+\frac{i}{2}\right)\right|=1 \text {. }
$$

$$
1+\delta \leqq \lim _{m \rightarrow \infty}\left|D F_{f_{m}}\left(\frac{1}{2}+\frac{i}{2}\right)\right|=\left|D F_{f_{\infty}}\left(\frac{1}{2}+\frac{i}{2}\right)\right|=1,
$$

which is a contradiction.

Finally, assume that $(* *)$ were to hold. Then

$$
l\left(D F_{f_{m}}\left(\frac{1}{2}+\frac{i}{2}\right)\right)<1-\delta,
$$

and proceeding in a manner similar to that above, we could assume $f_{m} \rightarrow f_{\infty}=\mathrm{id}_{\mathbf{R}}$, $A_{f_{m}} \rightarrow A=\mathrm{id}_{\mathrm{C}}$, and $F_{f_{m}} \rightarrow F_{f_{\infty}}=\mathrm{id}_{\mathrm{C}}$. Then

$$
\begin{aligned}
l\left(D F_{f_{m}}\left(\frac{1}{2}+\frac{i}{2}\right)\right) & =\min _{|h|=1}\left|D F_{f_{m}}\left(\frac{1}{2}+\frac{i}{2}\right) \cdot h\right| \\
& =\min _{|h|=1}\left|D A_{f_{m}}\left(B_{h_{m}}\left(\frac{1}{2}+\frac{i}{2}\right)\right) \cdot D B_{h_{m}}\left(\frac{1}{2}+\frac{i}{2}\right) \cdot h\right| \\
& =\left|A_{f_{m}}^{\prime}\left(B_{h_{m}}\left(\frac{1}{2}+\frac{i}{2}\right)\right)\right| l\left(D B_{h_{m}}\left(\frac{1}{2}+\frac{i}{2}\right)\right) .
\end{aligned}
$$

We infer that

$$
\lim _{m \rightarrow \infty} l\left(D F_{f_{m}}\left(\frac{1}{2}+\frac{i}{2}\right)\right)=l\left(D F_{f_{\infty}}\left(\frac{1}{2}+\frac{i}{2}\right)\right)=1,
$$

again a contradiction. Thus, it must be true that we are able to choose $L_{0}$ so that $L_{0}(H) \rightarrow 1$ as $H \rightarrow 1$.

We shall also need to know that $F_{f}$ is bilipschitz in the quasihyperbolic metrics of $\Omega_{0}$ and $\Omega_{1}$.

Lemma 9. Let $f: \mathbf{R} \rightarrow \mathbf{C}$ be a weakly H-quasisymmetric embedding as in Lemma 6, let $F_{f}$ be the Tukia extension of $f$, let $J=f(\mathbf{R})$ and let $C_{i}, i=0$ or 1 , be a component of $\mathbf{C} \backslash J$. Then there is a constant, $L_{1}=L_{1}(H) \geqq 1$, so that

$$
\frac{q(z, w)}{L_{1}} \leqq q_{C_{i}}\left(F_{f}(z), F_{f}(w)\right) \leqq L_{1} q(z, w)
$$

for all $z, w \in \Omega_{0}$ when $i=0$ or for all $z, w \in \Omega_{1}$ when $i=1$. Moreover, $L_{1}(H)$ may be chosen so that $L_{1}(H) \rightarrow 1$ as $H \rightarrow 1$. 
Proof. We carry out the proof for the case $F_{f}: \Omega_{0} \rightarrow C_{0}$. The other case is done in a similar manner. Let $u, v \in C_{0}$. Then

$$
q_{C_{0}}(u, v)=\inf _{\gamma^{*}} \int_{\gamma^{*}} \varrho_{C_{0}} d s
$$

where the infimum is extended over all rectifiable arcs $\gamma^{*}$ joining $u$ and $v$ in $C_{0}$.

Suppose we are able to establish that

$$
\frac{\varrho(z)}{l\left(D F_{f}(z)\right) L_{1}(H)} \leqq \varrho_{c_{0}}\left(F_{f}(z)\right) \leqq \frac{L_{1}(H) \varrho(z)}{\left|D F_{f}(z)\right|}
$$

where $L_{1}(H) \rightarrow 1$ as $H \rightarrow 1$.

Let $\gamma^{*}$ be a rectifiable arc joining $F_{f}(z)$ and $F_{f}(w)$ in $C_{0}$. Let $\gamma=F_{f}^{-1}\left(\gamma^{*}\right) \subset \Omega_{0}$. We see, using (10), that

$$
\begin{aligned}
q_{C_{0}}\left(F_{f}(z), F_{f}(w)\right) & \leqq \int_{\gamma^{*}} \varrho_{C_{0}} d s \\
& =\int_{F_{f} \circ \gamma} \varrho_{C_{0}} d s \\
& \leqq \int_{\nu}\left(\varrho_{C_{0}} \circ F_{f}\right)\left|D F_{f}\right| d s \\
& \leqq L_{1}(H) \int_{\gamma} \varrho d s .
\end{aligned}
$$

Taking the infimum over $\gamma$ gives the right-hand side of (9). Similarly,

$$
\begin{aligned}
q(z, w) & \leqq \int_{y} \varrho d s \\
& =\int_{F_{f}^{-1} \circ \gamma^{*}} \varrho d s \\
& \leqq \int_{\gamma^{*}}\left|D F_{f}^{-1}(w)\right| \varrho\left(F_{f}^{-1}(w)\right)|d w| \\
& =\int_{\gamma^{*}} \frac{1}{l\left[D F_{f}\left(F_{f}^{-1}(w)\right)\right]} \varrho\left(F_{f}^{-1}(w)\right)|d w| .
\end{aligned}
$$

Again using (10) we have

$$
q(z, w) \leqq L_{1}(H) \int_{\gamma^{*}} \varrho_{C_{0}}(w)|d w|
$$

and taking the infimum over $\gamma^{*}$ gives the left-hand side of (9). In the above we have used the fact that $F_{f}$ is a diffeomorphism off of $\mathbf{R}$.

Thus it suffices to show that (10) holds for each $z \in \Omega_{0}$. Fix $z=x+i y \in \Omega_{0}$. If $\varphi: \mathbf{R} \rightarrow \mathbf{R}$ is an increasing affine map, we denote by $\varphi$ as well the unique conformal affine map extending $\varphi$ to $\mathbf{C}$. Explicitly, we consider $\varphi$ defined by

$$
\varphi(w)=2 y w+(x-y)
$$

for $w \in \mathbf{R}$. Then $\varphi(0)=x-y$ and $\varphi(1)=x+y$. Let $\psi: \mathbf{C} \rightarrow \mathbf{C}$ be the conformal 
affine map such that $\psi(f(x-y))=0$ and $\psi(f(x+y))=1$. Set $g=\psi \circ f \circ \varphi$. We observe that $g$ is a normalized weakly $H$-quasisymmetric embedding. By [T 2, p. 92]

Thus

$$
F_{g}=F_{\psi \circ \rho \circ \varphi}=\psi \circ F_{f} \circ \varphi .
$$

$$
D F_{f}(x+i y)=D \psi^{-1}\left(F_{g}\left(\frac{1}{2}+\frac{i}{2}\right)\right) \cdot D F_{g}\left(\frac{1}{2}+\frac{i}{2}\right) \cdot D \varphi^{-1}(x+i y) .
$$

We note that both $D \psi^{-1}$ and $D \varphi^{-1}$ (which are constants) are similarity maps, $D \psi^{-1}$ multiplies by $[f(x+y)-f(x-y)]$ and $D \varphi^{-1}$ multiplies by $1 / 2 y$. Therefore we obtain

$$
D F_{g}\left(\frac{1}{2}+\frac{i}{2}\right)=\frac{2 y}{[f(x+y)-f(x-y)]} D F_{f}(x+i y) .
$$

Applying Lemma 8 to $g$ we arrive at

$$
\frac{|f(x+y)-f(x-y)|}{L_{0} 2|y|} \leqq l\left(D F_{f}(z)\right) \leqq\left|D F_{f}(z)\right| \leqq \frac{L_{0}|f(x+y)-f(x-y)|}{2|y|} .
$$

By Lemma 6,

$$
\frac{|2 y|^{\alpha}}{H} \leqq|f(x+y)-f(x-y)| \leqq H|2 y|^{\alpha} .
$$

Thus

$$
\frac{|y|^{\alpha}}{\left(2^{1-\alpha} H L_{0}|y|\right)} \leqq l\left(D F_{f}(z)\right) \leqq\left|D F_{f}(z)\right| \leqq \frac{\left(|y|^{\alpha} 2^{\alpha-1} H L_{0}\right)}{|y|} .
$$

Recall $\varrho_{C_{0}}\left(F_{f}(z)\right)=1 / \operatorname{dist}\left(F_{f}(z), J\right)$. Using $(7)$ we obtain

$$
\frac{1}{\left(M|y|^{\alpha}\right)} \leqq \varrho_{C_{0}}\left(F_{f}(z)\right) \leqq \frac{M}{|y|^{\alpha}} .
$$

Combining the above line with (12) we have

$$
\varrho_{C_{0}}\left(F_{f}(z)\right)\left|D F_{f}(z)\right| \leqq \frac{\left(M 2^{\alpha-1} H L_{0}\right)}{|y|} .
$$

Consequently, since $1 /|y|=\varrho(z)$, we have

$$
\varrho_{C_{0}}\left(F_{f}(z)\right) \leqq \frac{\left(2^{\alpha-1} H L_{0} M\right)}{\left|D F_{f}(z)\right|} \varrho(z) \leqq \frac{\left(2^{1-\alpha} H L_{0} M\right)}{\left|D F_{f}(z)\right|} \varrho(z)
$$

which is the right-hand side of (10), where $L_{1}=2^{1-\alpha} H L_{0} M$. Additionally,

$$
\begin{aligned}
\varrho_{C_{0}}\left(F_{f}(z)\right) & \geqq \frac{1}{\left(M|y|^{\alpha}\right)} \\
& \geqq \frac{1}{M}\left(\frac{1}{l\left(D F_{f}(z)\right)}\right)\left(\frac{1}{2^{1-\alpha} H L_{0}}\right)\left(\frac{1}{|y|}\right) \\
& =\frac{1}{\left(2^{1-\alpha} H L_{0} M\right)}\left(\frac{\varrho(z)}{l\left(D F_{f}(z)\right)}\right),
\end{aligned}
$$


which gives the left-hand side of (10), where again $L_{1}=L_{1}(H)=2^{1-\alpha} H L_{0} M$. Then (13) with (14) gives (10). We know that $2^{1-\alpha} H M \rightarrow 1$ as $H \rightarrow 1$. We apply Lemma 8 to see that $L_{0}$ may be chosen so that $L_{0}(H) \rightarrow 1$ as $H \rightarrow 1$. We conclude that $L_{1}(H) \rightarrow 1$ as $H \rightarrow 1$.

\section{Construction of the groups}

In order to produce the groups mentioned in the introduction, we essentially duplicate the steps involved in Tukia's construction. However, we have now acquired enough additional information to allow us to control the dilatation of the resulting groups. We remark that while we work in $\mathbf{R}^{n}$, all of the mappings involved fix the point $\infty$. Hence all of our results extend to $\overline{\mathbf{R}}^{n}$.

Let $G_{0}^{\prime}$ be the group consisting of all translations of $\mathbf{C}$ parallel to the real axis. Let $G_{1}^{\prime}$ be the group generated by $G_{0}^{\prime}$ and by the maps $\tilde{g}_{\lambda}: \mathbf{C} \rightarrow \mathbf{C}$, where $\tilde{g}_{\lambda}(z)=\lambda z$, for $\lambda>0$.

Fix $H>1$. Then Lemma 6 gives $f: \mathbf{R} \rightarrow \mathbf{C}$ such that $f$ is weakly $H$-quasisymmetric and satisfies (5). Let $F_{f}$ be the $K_{2}(H)$-quasiconformal Tukia extension of $f$ and let $J=f(\mathbf{R})$.

We define groups which depend on $F_{f}$, hence on $H$, as follows. Let

Then we have

$$
G_{0}^{\prime \prime}(H)=F_{f} \circ G_{0}^{\prime} \circ F_{f}^{-1}, \quad G_{1}^{\prime \prime}(H)=F_{f} \circ G_{1}^{\prime} \circ F_{f}^{-1} .
$$

Lemma 10. (a) The group $G_{0}^{\prime \prime}(H)$ acting on $\mathbf{C}$ is an $L_{2}$-Lipschitz group with respect to the usual metric, where $L_{2}=L_{2}(H)$ can be chosen so that $L_{2}(H) \rightarrow 1$ as $H \rightarrow 1$.

(b) $G_{1}^{\prime \prime}(H)$ is a $K_{3}$-quasiconformal group acting on $\mathbf{C}$. If the action of $G_{1}^{\prime \prime}(H)$ is restricted to a component $C_{i}, i=0$ or 1, of $\mathbf{C} \backslash J$, then $G_{1}^{\prime \prime}(H)$ is a Lipschitz group with respect to the quasihyperbolic metric $q_{C_{i}}$ of $C_{i}$. Furthermore, $K_{3}=K_{3}(H)$ can be selected so that $K_{3}(H) \rightarrow 1$ as $H \rightarrow 1$.

Proof of (a). We first show that there is an $L_{2} \geqq 1$ so that if $g \in G_{0}^{\prime \prime}(H)$ and $z \in \mathbf{C} \backslash J$ then

$$
\frac{1}{L_{2}} \leqq l\left(D_{g}(z)\right) \leqq\left|D_{g}(z)\right| \leqq L_{2} .
$$

Let $g \in G_{0}^{\prime \prime}(H)$. Let $z, w \in F_{f}\left(\Omega_{i}\right)=C_{i}, i=0$ or 1 . We have $g=F_{f^{\circ}} \circ g_{1} \circ F_{f}^{-1}$ where $g_{1} \in G_{0}^{\prime}$, hence is conformal. Thus

$$
\frac{1}{L_{1}^{2}} q_{C_{i}}(z, w) \leqq q_{C_{i}}(g(z), g(w)) \leqq L_{1}^{2} q_{C_{i}}(z, w),
$$

where we have used (9) and the fact that conformal maps are isometries with respect to $q_{\Omega_{i}}$. We see that $g$ is $L_{1}^{2}$-bilipschitz in the quasihyperbolic metric of $C_{i}$, where $L_{1}=L_{1}(H) \rightarrow 1$ as $H \rightarrow 1$. 
Let $z \in C_{i}$. Then $z=F_{f}(u+i v)$ for $u+i v \in \Omega_{i}$, whence $g(z)=F_{f}((u+a)+i v)$ for some $a \in \mathbf{R}$. An application of Lemma 7 gives

and

$$
\frac{|v|^{\alpha}}{M} \leqq \operatorname{dist}\left(F_{f}(u+a+i v), J\right) \leqq M|v|^{\alpha}
$$

$$
\frac{|v|^{\alpha}}{M} \leqq \operatorname{dist}\left(F_{f}(u+i v), J\right) \leqq M|v|^{\alpha} .
$$

As a consequence, we obtain

$$
\frac{1}{M^{2}} \leqq \frac{\operatorname{dist}\left(F_{f}(u+a+i v), J\right)}{\operatorname{dist}\left(F_{f}(u+i v), J\right)} \leqq M^{2} .
$$

From (16) we have

$$
\frac{1}{L_{1}^{2}} q_{C_{i}}(z, w) \leqq q_{C_{i}}(g(z), g(w)) \leqq L_{1}^{2} q_{C_{i}}(z, w) .
$$

This implies that

$$
\begin{aligned}
\frac{1}{L_{1}^{2} \operatorname{dist}(z, J)}=\lim _{w \rightarrow z} \frac{q_{C_{z}}(z, w)}{L_{1}^{2}|z-w|} & \leqq \varliminf_{w \rightarrow z} \frac{|g(z)-g(w)|}{|z-w|} \frac{q_{C_{t}}(g(z), g(w))}{|g(z)-g(w)|} \\
& \leqq \lim _{w \rightarrow z} \frac{|g(z)-g(w)|}{|z-w|} \frac{q_{C_{i}}(g(z), g(w))}{|g(z)-g(w)|} \\
& \leqq \lim _{w \rightarrow z} \frac{L_{1}^{2} q_{C_{i}}(z, w)}{|z-w|}=\frac{L_{1}^{2}}{\operatorname{dist}(z, J)} .
\end{aligned}
$$

If $z$ is a point where $g$ is differentiable, i.e., $z \notin J$, we see that

$$
\frac{1}{L_{1}^{2}} \frac{1}{\operatorname{dist}(z, J)} \leqq \frac{l\left(D_{g}(z)\right)}{\operatorname{dist}(g(z), J)} \leqq \frac{\left|D_{g}(z)\right|}{\operatorname{dist}(g(z), J)} \leqq L_{1}^{2} \frac{1}{\operatorname{dist}(z, J)} .
$$

So, at all points $z \in \mathbf{C} \backslash J$,

$$
\frac{1}{L_{1}^{2} M^{2}} \leqq l\left(D_{g}(z)\right) \leqq\left|D_{g}(z)\right| \leqq L_{1}^{2} M^{2} .
$$

It follows that (15) is true with $L_{2}=L_{1}^{2} M^{2}$.

We know $F_{f}$, hence $g$, is quasiconformal. Consequently, $g$ is differentiable a.e. and is absolutely continuous on lines. Since (15) holds a.e. in $\mathbf{C}$, it follows that $g$ is $L_{2}$-bilipschitz in C. Furthermore, $L_{2}=L_{2}(H) \rightarrow 1$ as $H \rightarrow 1$, and part (a) has been proved.

Proof of (b). Let $g \in G_{1}^{\prime \prime}(H)$. Then $g=F_{f} \circ g_{2} \circ F_{f}^{-1}$, where $g_{2} \in G_{1}^{\prime}$, hence is conformal. Since $F_{f}$ and $F_{f}^{-1}$ are $K_{2}$-quasiconformal, we see that $g$ is $K_{3}$-quasiconformal, where $K_{3}=K_{2}^{2}$. Hence $K_{3}=K_{3}(H) \rightarrow 1$ as $H \rightarrow 1$. An argument similar 
to the one used to obtain (16) in part (a) shows that

$$
\frac{q_{C_{t}}(z, w)}{L_{1}^{2}} \leqq q_{C_{i}}(g(z), g(w)) \leqq L_{1}^{2} q_{C_{i}}(z, w)
$$

for all $z, w \in C_{i}, i=0$ or 1 .

We now construct the desired groups. Fix $n \geqq 3$ and $\alpha>0$. We regard $\mathbf{R}^{n}$ as $\mathbf{R}^{2} \times \mathbf{R}^{n-2}=\mathbf{C} \times \mathbf{R}^{n-2}$ and write elements in $\mathbf{R}^{n}$ as $\tilde{x}=(z, t)$ with $z \in \mathbf{C}$ and $t \in \mathbf{R}^{n-2}$. Define maps $\hat{h}_{a}$ and $\hat{g}_{\lambda}$ of $\mathbf{R}^{n}$ for $a \in \mathbf{R}^{n}$ and $\lambda>0$ by

$$
\hat{h}_{a}(\tilde{x})=\tilde{x}+a, \quad \hat{g}_{\lambda}(z, t)=\left(\lambda z, \lambda^{\alpha} t\right) .
$$

We define groups $\hat{G}_{0}$ and $\hat{G}_{1}$ of affine mappings of $\mathbf{R}^{n}$ by

$$
\begin{aligned}
& \hat{G}_{0}=\left\{\hat{h}_{a}: a=\left(a_{1}, 0, a_{3}, \ldots, a_{n}\right), a_{i} \in \mathbf{R}\right\} \\
& \hat{G}_{1}=\hat{G}_{1}(\alpha)=\text { the group generated by } \hat{G}_{0} \text { and }\left\{\hat{g}_{\lambda}: \lambda>0\right\} .
\end{aligned}
$$

Then each $g \in \hat{G}_{1}$ is of the form $g(z, t)=\left(\lambda z+a, \lambda^{\alpha} t+b\right)$ for some $\lambda>0$, some $a \in \mathbf{R}$, and some $b \in \mathbf{R}^{n-2}$.

Now take $H>1$. Let $f: \mathbf{R} \rightarrow \mathbf{C}$ be a weakly $H$-quasisymmetric embedding as in Lemma 6 and let $\alpha=\alpha(H)$ be as in the same lemma. Then $\hat{G}_{1}=\hat{G}_{1}(\alpha)=\hat{G}_{1}(H)$. Set $\mathscr{F}=F_{f} \times \mathrm{id}_{\mathbf{R}^{n-2}}: \mathbf{C} \times \mathbf{R}^{n-2} \rightarrow \mathbf{C} \times \mathbf{R}^{n-2}$, where as before, $F_{f}$ is the Tukia extension of $f$. Define

$$
h_{a}=\mathscr{F} \circ \hat{h}_{a} \circ \mathscr{F}-1, \quad g_{\lambda}=\mathscr{F} \circ \hat{g}_{\lambda} \circ \mathscr{F}-1
$$

for $a \in \mathbf{R}^{n}$ and $\lambda>0$. Finally, set

$$
G_{0}(H)=\mathscr{F} \circ \hat{G}_{0} \circ \mathscr{F}^{-1}, \quad G_{1}(H)=\mathscr{F} \circ \hat{G}_{1} \circ \mathscr{F}^{-1} .
$$

We observe the following commutativity relations between $h_{a}$ and $g_{\lambda}$ :

We now have

$$
\left\{\begin{array}{l}
h_{\lambda a}=g_{\lambda} \circ h_{a} \circ g_{\lambda}^{-1}, \quad \text { for } a=\left(a_{1}, 0, \ldots, 0\right), \\
h_{\lambda \alpha_{b}}=g_{\lambda} \circ h_{b} \circ g_{\lambda}^{-1}, \text { for } b=\left(0,0, b_{3}, \ldots, b_{n}\right) .
\end{array}\right.
$$

Lemma 11. (a) The group $G_{0}(H)$ acting on $\mathbf{R}^{n}$ is an $L_{2}$-Lipschitz group with respect to the euclidean metric. Furthermore, $G_{0}(H)$ acts transitively on the invariant set $S=J \times \mathbf{R}^{n-2}$. Here $L_{2}=L_{2}(H)$ can be chosen to satisfy $L_{2}(H) \rightarrow 1$ as $H \rightarrow 1$.

(b) The group $G_{1}(H)$ is a $K_{4}$-quasiconformal group acting on $\mathbf{R}^{n}$. The action is transitive on $S$ and on each component of $\mathbf{R}^{n} \backslash S$. If the action of $G_{1}(H)$ is restricted to a component $D$ of $\mathbf{R}^{n} \backslash S$, then $G_{1}(H)$ is an $L_{3}$-Lipschitz group relative to the quasihyperbolic metric in D. Additionally, $K_{4}=K_{4}(H)$ and $L_{3}=L_{3}(H)$ can be assumed to satisfy $K_{4}(H) \rightarrow 1$ and $L_{3}(H) \rightarrow 1$ as $H \rightarrow 1$.

Proof of (a). Using Lemma 10(a) and the definition of $G_{0}(H)$, we see that $g \in G_{0}(H)$ is $L_{2}$-bilipschitz in the euclidean metric. Now if $S=J \times \mathbf{R}^{n-2}$, then $\hat{G}_{0}$ is transitive on $\mathscr{F}^{-1}(S)$, hence $G_{0}(H)=\mathscr{F} \circ \hat{G}_{0} \circ \mathscr{F}^{-1}$ is transitive on $S$. 
Proof of (b). Let $D$ be a component of $\mathbf{R}^{n} \backslash S$. Then $S$ and $D$ are invariant under the action of $G_{1}(H)=\mathscr{F} \circ \hat{G}_{1} \circ \mathscr{F}^{-1}$. It is evident that $\hat{G}_{1}$ acts transitively on $\mathscr{F}^{-1}(S)$, which implies that $G_{1}(H)$ is transitive on $S$. Furthermore, $\hat{G}_{1}$ is transitive on $\mathscr{F}^{-1}(D)$, so that the action of $G_{1}(H)$ is transitive on $D$.

An arbitrary $\hat{\mathrm{g}} \in \hat{G}_{1}$ may be written as $\hat{\mathrm{g}}=\hat{h}_{a} \circ \hat{\mathrm{g}}_{\lambda}$, for suitable $a$ and $\lambda$. This follows from (17). Accordingly, $g \in G_{1}(H)$ is of the form $g=h_{a} \circ g_{\lambda}$. To see that each $\left.g\right|_{D}=\left.h_{a} \circ g_{\lambda}\right|_{D}$ is bilipschitz with respect to the quasihyperbolic metric $q_{D}$, it suffices to show that $\left.g_{\lambda}\right|_{D}$ and $\left.h_{a}\right|_{D}$ are uniformly bilipschitz with respect to $q_{D}$.

For $\left.h_{a}\right|_{D}$ we have, by part (a),

$$
\frac{|\tilde{x}-\tilde{y}|}{L_{2}} \leqq\left|h_{a}(\tilde{x})-h_{a}(\tilde{y})\right| \leqq L_{2}|\tilde{x}-\tilde{y}|
$$

where $\tilde{x}, \tilde{y} \in D$ and $L_{2}=L_{2}(H) \rightarrow 1 \quad$ as $H \rightarrow 1$. Write $\tilde{x}=(z, t) \in \mathbf{C} \times \mathbf{R}^{n-2}$ and $z=F_{f}(u, v)$ with $(u, v) \in \mathbf{C}, v \neq 0$. Then

and

$$
\operatorname{dist}\left(h_{a}(\tilde{x}), S\right)=\operatorname{dist}\left(F_{f}\left(u+a_{1}, v\right), J\right),
$$

$$
\operatorname{dist}(\tilde{x}, S)=\operatorname{dist}(z, J)=\operatorname{dist}\left(F_{f}(u, v), J\right) .
$$

By Lemma 7 we know that

$$
\frac{|v|^{\alpha}}{M} \leqq \operatorname{dist}\left(F_{f}\left(u+a_{1}, v\right), J\right) \leqq M|v|^{\alpha},
$$

and

$$
\frac{|v|^{\alpha}}{M} \leqq \operatorname{dist}\left(F_{f}(u, v), J\right) \leqq M|v|^{\alpha},
$$

where $M=M(H)$ satisfies $M \rightarrow 1$ as $H \rightarrow 1$. We infer that

$$
\frac{1}{M^{2}} \leqq \frac{\operatorname{dist}\left(h_{a}(\tilde{x}), S\right)}{\operatorname{dist}(\tilde{x}, S)} \leqq M^{2} .
$$

This implies that

$$
\frac{1}{M^{2}} \varrho_{D}\left(h_{a}(\tilde{x})\right) \leqq \varrho_{D}(\tilde{x}) \leqq M^{2} \varrho_{D}\left(h_{a}(\tilde{x})\right),
$$

for all points $\tilde{x}$ in $D$.

Let $\beta$ be a rectifiable path joining $h_{a}(\tilde{x})$ to $h_{a}(\tilde{y})$ in $D$, and let $\gamma=h_{a}^{-1}(\beta)$. Then

$$
\begin{aligned}
q_{D}\left(h_{a}(\tilde{x}), h_{a}(\tilde{y})\right) & \leqq \int_{\beta} \varrho_{D}(\zeta)|d \zeta| \\
& \leqq \int_{\gamma} \varrho_{D}\left(h_{a}(\zeta)\right) L_{2}|d \zeta| \\
& \leqq L_{2} M^{2} \int_{\gamma} \varrho_{D}(\zeta)|d \zeta|
\end{aligned}
$$


where we have used part (a) and (18). Taking the infimum over $\gamma$ gives

$$
q_{D}\left(h_{a}(\tilde{x}), h_{a}(\tilde{y})\right) \leqq L_{2} M^{2} q_{D}(\tilde{x}, \tilde{y}) .
$$

This holds for all $h_{a} \in G_{0}(H)$, hence for $h_{a}^{-1}$, thus we see that

$$
\frac{q_{D}(\tilde{x}, \tilde{y})}{M^{2} L_{2}} \leqq q_{D}\left(h_{a}(\tilde{x}), h_{a}(\tilde{y})\right) \leqq M^{2} L_{2} q_{D}(\tilde{x}, \tilde{y}) .
$$

Consequently, $h_{a}$ is bilipschitz with respect to $q_{D}$.

We next consider the maps $g_{\lambda}$. Let $\tilde{x}=(z, t) \in D \subset \mathbf{C} \times \mathbf{R}^{n-2}, z=F_{f}(u, v)$ for $(u, v) \in \mathbf{C}$ with $v \neq 0$. We have

$$
\begin{aligned}
g_{\lambda}(\tilde{x}) & =\left(F_{f}\left(\lambda F_{f}^{-1}(z)\right), \lambda^{\alpha} t\right) \\
& =\left(g_{\lambda}^{*}(z), \lambda^{\alpha} t\right)
\end{aligned}
$$

where we set $g_{\lambda}^{*}(z)=F_{f}\left(\lambda F_{f}^{-1}(z)\right)$. Let

$$
\begin{aligned}
& d_{1}=\operatorname{dist}(\tilde{x}, S)=\operatorname{dist}(z, J), \\
& d_{2}=\operatorname{dist}\left(g_{\lambda}(\tilde{x}), S\right)=\operatorname{dist}\left(g_{\lambda}^{*}(z), J\right) .
\end{aligned}
$$

Since $z=F_{f}(u, v)$, by Lemma 7 we see that

$$
\frac{|v|^{\alpha}}{M} \leqq d_{1} \leqq M|v|^{\alpha},
$$

where $M=M(H) \rightarrow 1$ as $H \rightarrow 1$.

Now $g_{\lambda}^{*}(z)=F_{f}\left(\lambda F_{f}^{-1}(z)\right)=F_{f}(\lambda u, \lambda v)$, so

$$
\frac{|\lambda v|^{\alpha}}{M} \leqq d_{2} \leqq M|\lambda v|^{\alpha} .
$$

Consequently, we have

$$
\frac{\lambda^{\alpha}}{M^{2}} \leqq \frac{d_{2}}{d_{1}} \leqq \lambda^{\alpha} M^{2} .
$$

Observe that $D=C_{i} \times \mathbf{R}^{n-2}, i=0$ or 1 , where $C_{i}$ is a component of $\mathbf{C} \backslash J$. By definition, $g_{\lambda}^{*} \in G_{1}^{\prime \prime}(H)$. Therefore, applying Lemma 10 and (16) we conclude that $\left.g_{\lambda}^{*}\right|_{C_{i}}$ is $L_{1}^{2}$-bilipschitz in the quasihyperbolic metric of $C_{i}$, again where $L_{1}=$ $L_{1}(H) \rightarrow 1$ as $H \rightarrow 1$. For all $z, w \in C_{i}$ we infer that

$$
\frac{1}{L_{1}^{2}}\left(\frac{d_{2}}{d_{1}}\right) \leqq l\left(D g_{\lambda}^{*}(z)\right) \leqq\left|D g_{\lambda}^{*}(z)\right| \leqq L_{1}^{2}\left(\frac{d_{2}}{d_{1}}\right) .
$$

Using (20) we obtain

$$
\frac{\lambda^{\alpha}}{L_{1}^{2} M^{2}} \leqq l\left(D g_{\lambda}^{*}(z)\right) \leqq\left|D g_{\lambda}^{*}(z)\right| \leqq L_{1}^{2} M^{2} \lambda^{\alpha} .
$$

Let $h_{\lambda}^{*}: \mathbf{R}^{n-2} \rightarrow \mathbf{R}^{n-2}$ be the mapping defined by $h_{\lambda}^{*}(t)=\lambda^{\alpha} t$. Then $l\left(D h_{\lambda}^{*}(t)\right)=$ 
$\left|D h_{\lambda}^{*}(t)\right|=\lambda^{\alpha}$. Thus, for $\tilde{x}=(z, t) \in \mathbf{C} \times \mathbf{R}^{n-2}$, we have

which implies that

$$
g_{\lambda}(z, t)=\left(g_{\lambda}^{*}(z), h_{\lambda}^{*}(t)\right)
$$

$$
\frac{\lambda^{\alpha}}{L_{1}^{2} M^{2}} \leqq l\left(D g_{\lambda}(\tilde{x})\right) \leqq\left|D g_{\lambda}(\tilde{x})\right| \leqq L_{1}^{2} M^{2} \lambda^{\alpha},
$$

at each point $\tilde{x}$ in $D$.

It follows that

$$
\frac{q_{D}(\tilde{x}, \tilde{y})}{L_{3}} \leqq q_{D}\left(g_{\lambda}(\tilde{x}), g_{\lambda}(\tilde{y})\right) \leqq L_{3} q_{D}(\tilde{x}, \tilde{y}),
$$

where $L_{3}=L_{3}(H)=L_{1} M^{4}$. Clearly $L_{3} \rightarrow 1$ as $H \rightarrow 1$. Hence, $g_{\lambda}$ is bilipschitz with respect to the quasihyperbolic metric in $D$. Since any $g \in G_{1}(H)$ is of the form $\left.g\right|_{D}=\left.h_{a} \circ g_{\lambda}\right|_{D}$ we see that $\left.g\right|_{D}$ is $L_{4}=M^{2} L_{2} L_{3}$-bilipschitz with respect to the quasihyperbolic metric in $D$. By construction $L_{4}=L_{4}(H)$ satisfies $L_{4} \rightarrow 1$ as $H \rightarrow 1$.

Since $g_{\lambda}$ is a diffeomorphism, the linear dilatation of $g_{\lambda}$ at $\tilde{x}$ in $D$ is given by

$$
\frac{\left|D g_{\lambda}(\tilde{x})\right|}{l\left(D g_{\lambda}(\tilde{x})\right)} \leqq L_{1}^{4} M^{4}
$$

Therefore, for $\tilde{x} \in D$, the linear dilatation of $g$ is

$$
\begin{aligned}
\limsup _{r \rightarrow 0}\left(\frac{\max _{|\tilde{x}-\tilde{y}|=r}|g(\tilde{x})-g(\tilde{y})|}{\min _{|\tilde{x}-\tilde{y}|=r}|g(\tilde{x})-g(\tilde{y})|}\right) & \leqq L_{2}^{2} \frac{\left|D g_{\lambda}(\tilde{x})\right|}{l\left(D g_{\lambda}(\tilde{x})\right)} \\
& \leqq L_{2}^{2} L_{1}^{4} M^{4} .
\end{aligned}
$$

Now, $g$ is a diffeomorphism in $D$ with linear dilatation bounded by $L_{2}^{2} L_{1}^{4} M^{4}$. Then [V, 34.2] implies that $g$ is $K_{4}$-quasiconformal, with $K_{4}(H)=\left(L_{2}^{2} L_{1}^{4} M^{4}\right)^{n-1}$ where $K_{4} \rightarrow 1$ as $H \rightarrow 1$. Since $D$ was an arbitrary component of $\mathbf{R}^{n} \backslash S$, we see that $g$ is $K_{4}$-quasiconformal on $\mathbf{R}^{n} \backslash S$. Furthermore, each $g \in G_{1}(H)$ is absolutely continuous on lines in $\mathbf{R}^{n}$, and the $n$-measure of $S$ is zero. Hence [V, 34.6] implies that $G_{1}(H)$ is $K_{4}$-quasiconformal on $\mathbf{R}^{n}$.

The following lemma was first established by S. Rickman for $n=3$, and was later extended by Tukia. See [T 3] for details.

Lemma 12. The hypersurface $S=J \times \mathbf{R}^{n-2}$ constructed above is not quasiconformally locally flat. In particular, there is no quasiconformal homeomorphism $h$ of $\mathbf{R}^{n}$ onto itself such that $h\left(\mathbf{R}^{n-1}\right)=S$.

We now give the first of our results.

Theorem 13. The group $G_{0}(H)$ acting on $\mathbf{R}^{n}$ is an L-Lipschitz (hence $K=L^{2 n_{-}}$ quasiconformal) group with respect to the euclidean metric, where $L=L(H)$ can be chosen so that $L(H) \rightarrow 1$ as $H \rightarrow 1$. For no quasiconformal homeomorphism $h: \mathbf{R}^{n} \rightarrow \mathbf{R}^{n}$ is the conjugate $h \circ G_{0} \circ h^{-1}$ a Möbius group. 
Proof. We need only verify the last statement. Suppose $G_{0}=G_{0}(H)$ is of the form $h \circ G \circ h^{-1}$ where $G$ is a Möbius group and $h$ is quasiconformal. Using the Bieberbach theorems, Tukia showed that the orbit of a point under the action of such a conjugated group is the quasiconformal image of a hyperplane. Since the orbit of the origin under the action of our group $G_{0}$ is $S$, which is not the quasiconformal image of a hyperplane by Lemma 12, we have reached a contradiction.

We conclude with our second main result, whose proof is the same as in [T 3, p. 158] and is thus omitted.

Theorem 14. Let $H>1$. The group $G_{1}(H)$ acting on $\mathbf{R}^{n}$ is a $K$-quasiconformal group, where $K=K(H)$ can be chosen so that $K(H) \rightarrow 1$ as $H \rightarrow 1$. The action of $G_{1}(H)$ is transitive on $S=J \times \mathbf{R}^{n-2}$ and on each of the complementary domains $D$ of $\mathbf{R}^{n} \backslash S$. The domains $D$ are Jordan domains in $\mathbf{R}^{n}$ which are topologically, but not quasiconformally, equivalent to $B^{n}$.

Remarks. It can be shown that the group $G_{1}=G_{1}(H)$ is not isomorphic as a topological group to any Möbius group acting on $\overline{\mathbf{R}}^{m}$, for any $m>0$. See [T 3] for a proof. Using results of Martin, we conclude that every discrete subgroup of rank $n-1$ in the group $G_{0}(H)$ is not the quasiconformal conjugate of a Möbius group. Hence there exist $K(H)$-quasiconformal, elementary, discrete groups acting on $\mathbf{R}^{\boldsymbol{n}}$, with $K(H) \rightarrow 1$ as $H \rightarrow 1$. See [M, 3.8]. It is not known whether a non-elementary group fitting this description exists.

\section{References}

[A] AhLfors, L. V.: Lectures on quasiconformal mappings. - D. Van Nostrand Company, Inc. Princeton, New Jersey-Toronto-New York-London, 1966.

[BA] Beurling, A., and L. AhLfors: The boundary correspondence under quasiconformal mappings. - Acta Math. 96, 1956, 125-142.

[FS] Freedman, M. H., and R. Skora: Strange actions of groups on spheres. - To appear.

[GP] Gehring, F. W., and B. PalKa: Quasiconformally homogeneous domains. - J. Analyse Math. 30, 1976, 172-199.

[L] LeHTo, O.: Univalent functions and Teichmüller spaces. - Graduate Texts in Mathematics 109. Springer-Verlag, New York-Berlin-Heidelberg-London-Paris-Tokyo, 1987.

[M] MARTIN, G.: Discrete quasiconformal groups that are not the quasiconformal conjugates of Möbius groups. - To appear.

[MV] MCKemie, J., and J. VAALER: Weakly quasisymmetric embeddings. - To appear.

[S] Sullivan, D.: On the ergodic theory at infinity of an arbitrary discrete group of hyperbolic motions. - Riemann surfaces and related topics: Proceedings of the 1978 Stony Brook Conference, edited by I. Kra and B. Maskit, Annals of Mathematics Studies 97. Princeton University Press, Princeton, New Jersey, 1981, 465-496.

[T 1] TukIA, P.: On two-dimensional quasiconformal groups. - Ann. Acad. Sci. Fenn. Ser. A I Math. 5, 1980, 73-78.

[T 2] TukiA, P.: Extension of quasisymmetric and Lipschitz embeddings of the real line into the plane. - Ann. Acad. Sci. Fenn. Ser. A I Math. 6, 1981, 89-94. 
[T 3] TUKIA, P.: A quasiconformal group not isomorphic to a Möbius group. - Ann. Acad. Sci. Fenn. Ser. A I Math. 6, 1981, 149-160.

[TV1] TukIA, P., and J. VÄISÄLÄ: Quasisymmetric embeddings of metric spaces. - Ann. Acad. Sci. Fenn. Ser. A I Math. 5, 1980, 97-114.

[TV2] TUkIA, P., and J. VÄISÄLÄ: Extension of embeddings close to isometries or similarities. Ann. Acad. Sci. Fenn. Ser. A I Math. 9, 1984, 153-175.

[V] VÄISÄL ̈̈, J.: Lectures in $n$-dimensional quasiconformal mappings. - Lecture Notes in Mathematics 229. Springer-Verlag, Berlin-Heidelberg-New York, 1971.

University of Missouri-Rolla

Department of Mathematics and Statistics

Rolla, Missouri 65401-0249

U.S.A.

Received 5 March 1986 